\title{
Educación inclusiva en las instituciones de educación superior: narrativas de estudiantes con discapacidad
}

\author{
Inclusive education in higher education institutions: narratives of \\ students with disabilities
}

\section{Resumen}

En este artículo se presentan los resultados parciales de una tesis doctoral vinculada a un proyecto de investigación financiado por el Ministerio de Economía y Competitividad, en el que se pretendía dar a conocer los facilitadores y obstaculizadores que los estudiantes con discapacidad se encontraron durante su estancia en las instituciones de enseñanza superior. A partir del método biográfico-narrativo se pretendía exponer el punto de vista de los estudiantes con discapacidad, identificando y explicando las barreras y ayudas que estos jóvenes vivenciaron en su trayectoria universitaria. Para la recogida de información se ha hecho uso de diversos instrumentos biográficos como son entrevistas, fotografías o líneas de vida. En concreto, los resultados de este trabajo se centran en las principales barreras y ayudas que este colectivo identificó en la universidad a nivel institucional. Entre las principales conclusiones obtenidas podemos destacar cómo la normativa existente no garantiza una educación de calidad, la importancia de los servicios de apoyo para la orientación y asesoramiento durante sus trayectorias o, por ejemplo, el personal de administración y servicio como factor resiliente.

\section{Palabras clave}

Enseñanza superior, discapacidad, metodología biográfico-narrativa, inclusión, educación.

\section{Abstract}

This paper presents the partial results of a doctoral thesis linked to a research project financed by the Ministry of Economy and Competitiveness, which aims at divulging the enablers and significant obstacles that students with disabilities encountered during their stay in institutions of higher education. Using a biographical narrative the points of view of the students with disabilities is exposed identifying and explaining the barriers and aids these young people experienced at the University. For the collection of information, various instruments such as biographical interviews, photographs and lifelines were used. Specifically, the results achieved of this work focuses to know on the main barriers and help that this group identified at the institutional level at the University. The main conclusions highlight that quality education is not guaranteed by the current legislation and that support services for guidance and advice during their careers or, for example, the administration and service are of great importance as they serve as resilient factor.

\section{Keywords}

Higher education, disability, biographical narrative methodology, inclusion, education.

\author{
Almudena Cotán Fernández \\ <almudena.cotan@uiı.es> \\ Universidad Internacional Isabel I
}

Para citar:

Cotán, A. (2017): "Educación inclusiva en las instituciones de educación superior: narrativas de estudiantes con discapacidad". Revista Española de Discapacidad, 5 (I): 43-6I.

Doi: <https://doi.org/IO. 5569/23405 I04.05.01.03>

Fecha de recepción: 07-09-2016 Fecha de aceptación: 10-03-20I7 


\section{Introducción}

Uno de los principales retos a los que actualmente se enfrenta la educación superior es garantizar una educación de calidad en igualdad a todos los estudiantes, con independencia de sus capacidades y reconociendo su derecho a la participación en los procesos de aprendizaje (Alba y Zubillaga, 20I 2; Cobos y Moreno, 20I4).

Desde el Real Decreto Legislativo I/20I3 por el que se aprobó el Texto Refundido de la Ley General de derechos de las personas con discapacidad y de su inclusión social, la accesibilidad se reconoce como el derecho que tiene la persona con discapacidad a participar de forma plena en todos los ámbitos en las mismas condiciones de igualdad que el resto de las personas. Para ello, tanto el Gobierno como los poderes públicos han de adoptar las medidas que consideren oportunas para garantizar la accesibilidad universal y la igualdad de oportunidades en los diferentes entornos, servicios, transportes, etc. (art.22).

Por su parte, para que se cumplan las condiciones básicas de accesibilidad y no discriminación, el Gobierno ha de regular las condiciones básicas que garanticen la igualdad de oportunidades en los diferentes niveles, al menos, en los siguientes aspectos (art.23):

- Accesibilidad en los edificios y entornos, suprimiendo barreras en las instalaciones y adaptando equipos e instrumentos, señalizándolos.

- Condiciones más favorables en el acceso, participación y utilización de los recursos dentro de cada ámbito o área.

- Apoyos complementarios (ayudas económicas, tecnológicas, personales, etc.).

- Creación de normas internas en las empresas o centros con el fin de eliminar las situaciones de desventaja o discriminación.

- Creación de planes y calendarios para implantar las exigencias de accesibilidad.
- Dotación de recursos humanos y materiales para la promoción de la accesibilidad y la no discriminación en el ámbito del que se trate (comunicación, transporte, edificios, etc.).

En este sentido, el Libro Verde de la Discapacidad (IMSERSO, 2002) define el concepto de accesibilidad en términos como "acceso, acción de llegar y acercarse, entrada o paso” (CRUE y Fundación ONCE, 20I4: 29). Por su parte, Peralta (2007: 47) indica que "el Diseño para Todos y la Accesibilidad Universal significa responder de manera adecuada a los diferentes retos que plantea una sociedad cada día más compleja y heterogénea, y con usuarios de características y potencialidades diversas”.

Desde nuestro punto de vista, estamos de acuerdo con autores como Peralta (2007) y Solla (2013) al considerar que el concepto de accesibilidad no sólo debería aludir a las personas con discapacidad, sino a toda la ciudadanía, donde la supresión de barreras en todos los espacios, productos o servicios sea una condición indispensable para que se cumpla el principio de igualdad de oportunidades.

En este sentido, CRUE y Fundación ONCE (20I4) indican que los principios de accesibilidad en las instituciones de educación superior han de basarse en el diseño para todas las personas centrándose en dos esferas: la organización y la docencia.

Desde el punto de vista organizacional y bajo el prisma del modelo social de discapacidad y del modelo de diversidad funcional, las transformaciones en este ámbito (estrategias, recursos, evaluación, etc.) han de basarse en el contexto y nunca en la persona tal y como propugnaba el modelo médico de discapacidad. Relacionado con esto, podemos encontrar trabajos como los de Moriña et al. (20I4), quienes aseguran que las tutorías son esenciales para asegurar un correcto proceso de aprendizaje así como la adaptación con antelación de los materiales y recursos que se van a utilizar durante la materia y la adaptación de las pruebas de evaluación (tiempo o recursos, entre otros). 
Desde la visión de los docentes, CRUE y Fundación ONCE (20I4) aluden a los tres principios del diseño universal de aprendizaje: múltiples medios de representación, múltiples medios de expresión y múltiples medios de compromiso (Guasch y Hernández, 20 I I). Asimismo, trabajos como los de Cobos y Moreno (20I4), Moriña (2OI 5), Moriña, et al. (201 5), Sánchez Palomino (2009) o Vander Kloet (2OI 5 ) ratifican esta idea indicando que si el profesorado facilitara con antelación los materiales con los que van a trabajar y mostraran una actitud abierta, reflexiva y positiva hacia sus necesidades, se optimizarían los procesos de aprendizaje del alumnado con discapacidad ayudando, además, a que este colectivo supere de forma exitosa el proceso de aprendizaje de la materia.

Sin embargo, en la actualidad las instituciones de educación superior ofrecen respuestas de diversa naturaleza para implantar de manera efectiva el principio de igualdad de oportunidades y de accesibilidad entre su alumnado (Castro de Paz y Abad Morillas, 2009). El acceso de los estudiantes con discapacidad a la educación superior ha de regirse bajo los principios del diseño universal donde los espacios físicos, transportes, comunicación, tecnologías de la información y comunicación, espacios virtuales u otros servicios sean extensibles a este colectivo en las mismas condiciones que el resto de la comunidad universitaria (Ferreira et al., 20I4; Pujolà, 2007).

Por ello, consideramos que para que se realice de una manera efectiva y óptima el proceso de igualdad en el acceso del alumnado universitario, se requiere de la implantación de programas de apoyo, orientación y asesoramiento, favoreciendo por lo tanto el proceso de integración y de inclusión de las personas con discapacidad a los estudios universitarios.

Siguiendo los datos ofrecidos por la Fundación Universia (2016), de las 76 universidades españolas la mayoría de ellas ofrecen algún servicio de apoyo o de atención al alumnado con discapacidad, pudiendo ser a través de servicios, programas específicos o mediante otras estructuras durante su acceso, ingreso y estancia en la universidad. En este sentido, resulta llamativo que el $89,4 \%$ de las universidades recogidas en la Guia de Apoyo a las Necesidades Educativas del Estudiante con Discapacidad (Fundación Universia, 20I6) realice adaptaciones curriculares, eximan del pago de las tasas académicas $(70 \%)$, realicen un procedimiento de acogida e inscripción de nuevo ingreso especializado para el estudiante con discapacidad (70\%) o construyan y adapten sus edificios y espacios bajo los principios de accesibilidad (95\%). Sin embargo, tan sólo el $60 \%$ de estas instituciones ofrece asesoramiento específico a personas con discapacidad mental o realiza acciones de fomento hacia el emprendimiento $(35 \%)$.

En relación con ello, podemos citar el trabajo de investigación de Castro de Paz y Abad Morillas (2009), cuyos datos reflejan las demandas de los estudiantes con discapacidad destacando la coordinación entre los niveles pre-universitarios y la universidad, la regulación de los servicios de orientación y de apoyo a la discapacidad dentro del contexto universitario, la creación de un servicio de asesoramiento 'proactivo' que gestione y optimice la información, los recursos y los apoyos existentes y que las actuaciones de éste se encuentren dirigidas tanto al alumnado como al profesorado, así como una mejor coordinación entre la universidad y las distintas administraciones e instituciones cuyo fin sea mejorar la vida de las personas con discapacidad.

Por otro lado, también nos encontramos con investigaciones como las de Moriña et al. (20I5) quienes, bajo la perspectiva de los estudiantes con discapacidad, identifican cuatro peticiones: I. información y orientación para mejorar y facilitar su vida universitaria, 2. docentes informados y formados para dar respuesta a sus necesidades educativas y que favorezcan su inclusión en las aulas, 3. entornos universitarios e infraestructuras accesibles y 4 . una universidad formada y atenta a las necesidades del colectivo de alumnos con discapacidad.

No obstante, y de acuerdo con autores como Moriña (20I 5), Peralta (2007) y Tolrá 
(2002), podemos afirmar que las instituciones de educación superior han ido iniciando y realizando esfuerzos cada vez mayores para elaborar y continuar los programas de apoyo y orientación hacia el colectivo de estudiantes con discapacidad para su inclusión en la vida universitaria. Pero igualmente es cierto, tal y como hemos ido señalando en líneas anteriores, que la consolidación de los principios de igualdad, inclusión y no discriminación de este colectivo es un proceso lento y lejano. Además, tal y como indican CRUE y Fundación ONCE (2014: 30), "la información y sensibilización de agentes presentes en el proceso educativo debe hacerse desde la premisa de que los derechos de las personas con discapacidad son una cuestión de ciudadanía y no de solidaridad".

Es por ello que desde este trabajo nos centraremos en "identificar, describir y explicar las barreras y ayudas que un grupo de estudiantes con discapacidad perciben en la universidad como institución" a partir de una metodología biográfico-narrativa.

\section{Metodología}

Los resultados presentados en este artículo se encuadran dentro de una tesis doctoral vinculada a un proyecto $\mathrm{I}+\mathrm{D}+\mathrm{i}$ financiado por el Ministerio de Economía y Competitividad (ref. EDU2010-16264). Este proyecto ha sido desarrollado por un equipo de investigación interdisciplinar de la Universidad de Sevilla de diferentes áreas y campos de conocimientos (Ciencias de la Educación, Ciencias Económicas, Ciencias de la Salud y Ciencias Experimentales). Su propósito principal fue estudiar desde la perspectiva de los estudiantes con discapacidad las barreras y ayudas que identificaron en su acceso, trayectoria y resultados en la Universidad de Sevilla, siempre atendiendo a sus narraciones sobre sus propias vivencias y experiencias.
Por ello, fruto de nuestra estrecha vinculación con este estudio y motivados por los conocimientos y resultados de la misma, procedimos a dar sentido y origen al trabajo que actualmente estamos presentando, planteándonos cuestiones como: ¿cuáles son las principales barreras y ayudas con las que los estudiantes con discapacidad se encuentran en la universidad?, ¿cómo se enfrentan a ellas?, ¿cómo participan en la vida universitaria?, ¿cómo se sienten ante situaciones de segregación o inclusión?, etc.

Este trabajo de investigación complementa otros trabajos previos sobre esta temática (Borland y James, I999; Doughty y Allan, 2008; Jacklin et al. 2007; Moriña, 20I 5; Moriña et al. 2016; Murray et al. 20I I; Sánchez Palomino, 2009, 20I I; Prowse, 2009; Tinklin y Hall, I999), haciendo hincapié en las vivencias subjetivas y narraciones de los estudiantes con discapacidad a través de una metodología biográficonarrativa. Entre las principales potencialidades de este estudio cabe resaltar el propio objetivo del estudio centrado en analizar tanto los procesos obstaculizadores como facilitadores que los estudiantes con discapacidad identifican en la universidad como institución y, por otro lado, la propia metodología centrada en hacer oír la voz de los alumnos en el contexto universitario "sin silenciar su subjetividad" (Moriña y Molina, 20I : 26), ya que la mayoría de investigaciones previas sobre esta temática se han basado en estudios bajo un paradigma cuantitativo o mixto.

En este sentido, consideramos que, bajo el planteamiento de nuestro estudio, la metodología de investigación seleccionada es la adecuada ya que, partiendo de sus voces, nos permite conocer las relaciones entre la vida de las personas y las situaciones o contextos donde se generan para, de este modo, tener una visión globalizada de la misma (Holstein y Gubrium, 20I2), así como establecer las relaciones casuales y las conexiones existentes entre los diversos acontecimientos que pueden suceder en la vida de las personas (Coninck y Godard, I 998), siguiendo un orden de temporalidad en la aparición de los acontecimientos con un enfoque 
de tipo balístico que separa claramente el pasado del futuro pero que establece una relación entre ambos.

Bajo este enfoque metodológico nos acercaremos al tema de estudio a través de las percepciones y vivencias que los estudiantes con discapacidad tienen de sus experiencias universitarias en el ámbito institucional (en los distintos servicios universitarios, recibimiento en la universidad, accesibilidad de los espacios, etc.) para dar respuesta al primer objetivo de la investigación: "identificar, describir y explicar las barreras y ayudas que un grupo de estudiantes con discapacidad perciben en la universidad como institución”.

Para ello y, partiendo del mismo, pretendemos analizar de forma general los diferentes mecanismos de acceso a la universidad. Desde este punto de vista, queremos estudiar si al acceder a la universidad este alumnado se tiene que enfrentar a algún tipo de obstáculo o, todo lo contrario, con ninguno. También nos planteamos con este objetivo responder a la cuestión de qué ocurre durante el transcurso de los estudios universitarios. Es decir, queremos conocer las limitaciones y facilidades que el colectivo que conforma la muestra de este estudio identifica en la institución universitaria durante su estancia en la misma.

\subsection{Recogida de datos}

Para dar respuesta a los objetivos de la investigación y, en concreto, al objetivo presentado en este artículo, la recogida de información se ha organizado en torno a dos áreas de conocimiento: Ciencias e Ingeniería (un participante) y Ciencias Sociales y Jurídicas (dos participantes).

Los hallazgos que presentamos en este artículo han sido recogidos durante cinco años (desde 20I0 hasta 20I5) a través de diversas técnicas e instrumentos elaborados para la investigación basándonos, principalmente, en entrevistas biográficas. Asimismo, se ha hecho uso de otros instrumentos como son entrevistas focalizadas, entrevistas semi-estructuradas, fotografías, auto-informes, línea de vida, diario de campo de la investigadora, notas de campo de la investigadora, observaciones, correos electrónicos y llamadas telefónicas así como la técnica "Un día en la vida de...".

En concreto, los resultados presentados en este artículo se obtuvieron a través de las siguientes técnicas:

- Entrevistas biográficas o en profundidad: estas entrevistas, que se caracterizarán por ser no estructuradas, permitirán profundizar de una forma detallada en las historias de vida de la trayectoria universitaria de los estudiantes con discapacidad.

- Entrevistas focalizadas: serán entrevistas que girarán en torno a un aspecto muy concreto de la trayectoria universitaria de los participantes en esta investigación. Esta técnica servirá para recoger incidentes críticos de la vida universitaria de los participantes en el estudio.

- Auto-informe: a través de este instrumento, el propio participante narra por escrito, en primera persona, los aspectos que considera más significativos para el tema de estudio propuesto.

- Línea de vida o timeline: esta técnica supone una revisión globalizada de los momentos más importantes en la experiencia de los participantes. Para ello, realizamos un eje de coordenadas en la línea, donde en el eje de abscisas (X) situaremos el año académico y en el eje de ordenadas (Y) colocaremos una puntuación entre el o y el ıo. El alumno en cada curso académico ha de definir un suceso o experiencia relevante en su historia otorgándole una posición. De esta forma, obtenemos una línea con ascensos y descensos que nos ofrecerán una visión gráfica y globalizada de su trayectoria universitaria.

- “Un día en la vida de...": con esta técnica hemos pasado una tarde o una mañana con el participante, analizando todas las barreras y ayudas que el estudiante 
encuentra en su experiencia universitaria desde que sale de su casa hasta que llega, con el fin de ver lo que ocurre en su día a día.

No obstante, debemos indicar que las técnicas empleadas han sido similares en todos los casos, sin embargo no todas se han llevado a cabo con todos los participantes puesto que significaba redundar la información o no tenían disponibilidad para poder realizarla.

\subsection{Participantes}

En esta investigación la muestra ha sido obtenida a través de la primera fase del mencionado proyecto a través del cual obtuvimos una muestra de tres alumnos con diversas discapacidades.

Para la selección de los participantes de nuestro estudio nos hemos basado en el procedimiento establecido por Goetz y LeCompte (I988), denominado "selección basada en criterios", donde la selección se encuentra determinada previamente por unos criterios establecidos por la investigadora. En este sentido, y siguiendo a Moriña (2003), Patton (I987) y Pujadas (2002), hemos realizado un muestreo selectivo atendiendo a unos rasgos, criterios y características relevantes, en concreto siete, para la selección de los tres participantes: estudiante con discapacidad, accesibilidad, participación, disponibilidad para participar, relevancia, voluntariedad e interés o necesidad.

De los tres participantes, dos son hombres y una mujer, cuyas titulaciones son variadas (Derecho, Ingeniería y Periodismo). En líneas generales, la edad de los participantes (en el momento de la recogida de información) es de 28 años de media, con una permanencia en la universidad de diez, seis y cuatro años. Por último, en cuanto a la discapacidad, y siguiendo la clasificación realizada por la Universidad de Sevilla, estos participantes presentan discapacidad física (parálisis cerebral y enfermedad de Duchenne) y visual (ceguera).

\subsection{Análisis de los datos}

Este apartado del trabajo se ha abordado desde una doble perspectiva. Por un lado, para la elaboración de cada historia se partió de un análisis narrativo (Goodley et al. 2004).

Por otro lado, el análisis estructural (Riessman, 2008), partiendo de los antecedentes inmediatos de esta investigación, se ha realizado a través de un sistema de codificación propuesto por Miles y Huberman (I994), que ha sido modificado tras la recogida de datos en la investigación "ya que el grupo final de categorías no está totalmente predeterminado sino que está compuesto por datos de acuerdo con los significados de categorías” (McMillan y Schumacher, 2005: 486).

A través de la codificación temática creada para analizar los datos obtenidos de las entrevistas, línea de vida, fotografías, notas de campo, etc., pretendemos conocer de forma transversal lo que los participantes cuentan y narran de su experiencia universitaria. La lista final de categorías ha quedado organizada en torno a seis criterios: I. información general, 2. barreras institucionales, 3 . ayudas institucionales, 4. barreras de aula, 5. ayudas de aula y 6 . prospectivas.

Los datos que no han sido incluidos en ninguna categoría han sido revisados en reiteradas ocasiones para asignarle o crear una nueva. Para codificar todos estos datos hemos utilizado el programa informático de análisis de datos MAXQDA זo.

Concretamente, en este trabajo presentamos los datos obtenidos a través del segundo análisis propuesto, el análisis estructural centrándonos en las tres primeras categorías.

\subsection{Consideraciones éticas}

Desde el punto de vista de nuestro trabajo de investigación y, en concreto, desde la investigación biográfico-narrativa, se plantea una serie de consideraciones éticas (Hammersley 
y Atkinson, 2007; Opazo, 20I I; Pacheco, 20I 2; Richaud, 2007; Sikes, 2006; Stutchbury y Fox, 2009; Vain, 20II) que deben ser tenidas en cuenta. Por ello, en nuestro trabajo de investigación todos los participantes dieron por escrito su consentimiento informado para formar parte del estudio. A estos estudiantes se les garantizó en todo momento el anonimato y confidencialidad de toda la información recogida, danto la opción de suprimir o modificar nombres, lugares o instituciones que el estudiante considerase que le pudiera identificar.

Durante todo el proceso del estudio el estudiante ha tenido acceso a la información recopilada, pudiendo modificarla o suprimirla si así lo considerase oportuno. Además, se les informó de que en el caso de no querer continuar adelante con el estudio una vez iniciado, sus datos no serían tenidos en cuenta para el análisis, pasando a ser destruidos. Otra cuestión que se trató fue la coparticipación del estudiante como coinvestigador durante la investigación, participando tanto en la toma de decisiones como en la elaboración y diseño de las técnicas de recogida de datos.

\section{Resultados}

En la mencionada tesis doctoral se ha realizado un análisis completo sobre las principales barreras y ayudas que los estudiantes con discapacidad identificaron en la universidad en diversas áreas: profesorado, proyectos docentes, metodología o compañeros, entre otros. En líneas generales, los tres participantes se centraron principalmente en destacar las carencias y necesidades que ellos han experimentado en la Universidad de Sevilla, más que en los aspectos positivos y ayudas que ésta les ofrece.

Sin embargo, para avanzar hacia espacios accesibles e inclusivos en las instituciones de educación superior, en este trabajo sólo vamos a presentar los resultados relativos a la universidad como institución centrándonos en las principales barreras y ayudas que los estudiantes con discapacidad identifican en la universidad, facultades, vicerrectorado, secretaría, etc.

\section{1. ¿Es la universidad un espacio accesible?}

Uno de los problemas principales que los alumnos identificaron a lo largo de su trayectoria universitaria es la laguna normativa existente en la universidad y, como consecuencia, las subyacentes trabas organizativas y actitudinales que de ellas se derivan. La normativa avala, resguarda y protege los derechos de los estudiantes, sin embargo, son desconocidos por la comunidad universitaria, incluyendo al profesorado. Es necesario, por tanto, su difusión efectiva para que pueda cumplirse. Como consecuencia, este desconocimiento afecta a la organización interna y al quehacer diario de cada facultad pues, ante estos temas, las respuestas no se rigen por la normativa.

El acceso a la universidad también supuso para estos estudiantes un momento complejo ocasionado por la desorientación y la desinformación. Precisamente, una de las ayudas mejor valoradas por estos estudiantes con discapacidad es la orientación y apoyo recibido por parte de los profesionales de la universidad que realizaron las adaptaciones que se consideraban pertinentes en los exámenes. Sin embargo, una de las mayores trabas y barreras con la que se encontraron es con la escasa continuidad de estas adaptaciones y la falta de información que recibieron sobre ayudas, becas, ofertas de trabajo y cursos de formación. Por otro lado, resaltar la experiencia de Andrés, quién vivió este trámite como caótico puesto que, bajo su experiencia, no había ni personal suficiente para responder a las necesidades del alumnado ni recursos suficientes para poder elaborar las pruebas de selectividad. Desde su punto de vista, para él esta experiencia resultó ser segregadora con respecto al resto de compañeros, ya que lo ubicaron en un aula diferente.

"Yo entré en la universidad en el año 2006, estoy muy descontento con el servicio de 
selectividad y de acceso porque, sinceramente, eso es un caos tremendo, ahí no hay control ninguno, faltan profesores, se distribuye a la gente por discapacidades en diferentes salas, solamente hay una persona que es profesor de la materia que te estás examinando y si tienes alguna duda es prácticamente imposible solucionarla" (Andrés, primera sesión, línea de vida).

"La verdad es que mi experiencia en selectividad fue bastante positiva. Meses antes, me evaluaron y dije el tipo de necesidades académicas y adaptaciones que necesito para hacer examen. Yo iba un poco con miedo a hacer el examen de selectividad, no sé... como me lo hacían aparte, iba un poco separado del grupo de mis compañeros e iba un poco asustado, pero con el trato que recibí el primer día pues... fue maravilloso. Hay un grupo especializado en personas con discapacidad y allí sí me atendieron bastante bien, me apoyaron en todos los recursos que necesitaba y eso. Pero ya, conforme salí de allí, no te orientaban sobre los tipos de apoyo que tiene la facultad" (Rafa, primera sesión, entrevista biográfica).

No obstante, hay quienes reclamaron la existencia de algún organismo, entidad, departamento o figura de apoyo que les ayudara durante su primer año, ya que afirmaron que se sentían desamparados y dependientes de la buena voluntad del personal de la universidad. Por ejemplo, Rafa explicó el desconocimiento, desinformación y desorientación que sufrió al acceder a la universidad a la hora de realizar el primer año de matriculación en la misma.

"Echo en falta un servicio de orientación. No orientan bien a los profesores sobre las necesidades de las personas con algún tipo de discapacidad cuando se encuentran en sus clases, ni en el acceso a la universidad. No tienes ningún tipo de orientación académica" (Rafa, primera sesión, entrevista biográfica).

Un segundo problema identificado es la falta de algún organismo o entidad que satisficiera sus necesidades durante su estancia en la universidad ya que explicaron que las ayudas que recibían dependían de la buena voluntad del personal de la universidad: las ayudas de los profesores, los conserjes, biblioteca... pero no porque existiera ningún organismo ni normativa. No obstante, señalaron esa buena voluntad como algo positivo ya que indicaron un cambio de mentalidad y un aumento de sensibilidad ante sus demandas. Unido a esto, uno de los participantes, Andrés, indicó cómo en cierta medida sus necesidades fueron satisfechas y cómo el servicio de conserjería se encontraba muy comprometido con su trabajo mejorando curso tras curso y convirtiéndose en el pilar más importante para él dentro de la universidad. Entre sus funciones principales destacaron la reserva de espacios, las ayudas en determinadas adaptaciones arquitectónicas y su enorme empatía con y hacia ellos para solucionar los problemas de forma eficaz, satisfactoria y con poca o ningún tipo de burocracia.

"Yo con la gente de conserjería estoy muy contento porque el servicio ha mejorado y sigue mejorando cada año más, pero en general, no solamente conmigo. Son gente que están muy comprometidos con su trabajo y que te ayudan” (Andrés, primera sesión, entrevista biográfica).

Respecto a los servicios de biblioteca, los tres estudiantes se encontraron muy satisfechos y, pese a que la cafetería normalmente suele ser un servicio externo a la institución, reconocen sentirse queridos e integrados dentro de estos espacios recibiendo un apoyo importante por parte de los trabajadores de la misma.

"No me puedo quejar en absoluto de los profesionales de la facultad. Todos me han tratado muy bien a lo largo de todos estos años, pero concretamente me sorprende el personal de la cafetería, ya que son trabajadores muy eficientes dándose cuenta de la situación rápidamente. Digo esto porque el primer día que entré en la cafetería, pedí una cañita para tomarme el café y no había y, desde entonces, nunca han faltado cañitas en la cafetería” (Marper, auto-informe). 
Otra dificultad señalada por los estudiantes es la falta de información que la universidad no facilitaba ni proporcionaba a los profesores sobre su discapacidad, no permitiendo al profesorado organizar y estructurar el currículo con antelación. Precisamente, esta información es recogida durante el periodo de matriculación, indicándose si se tiene o no discapacidad, el tipo y el grado y aseguran que dicha información no llega al profesorado y, peor aún, no se sabe exactamente a dónde va a parar y qué utilidad se le da.

Uniéndose a esta queja, el alumnado con discapacidad visual reclama la creación de un servicio interno que pudiera adaptar y/o transcribir los apuntes o que realice programas especiales, en relación al trabajo que vienen haciendo organismos externos como es el caso de la ONCE. Por ello, los estudiantes opinaron que el profesorado debería dejar sus materiales por adelantado para que pudieran adaptarse a tiempo y no dejarlos paulatinamente durante el curso, ya que en el caso de los estudiantes con discapacidad les dificultaba llevar el ritmo de las clases puesto que, en primer lugar, los estudiantes debían adquirir los apuntes; en segundo lugar, éstos tenían que llevarlos a otro organismo y, en tercer lugar, había que esperar a que estos apuntes se transcribieran para poder recibirlos. Esto conllevaba un largo período temporal en el cual los estudiantes no podían estudiar y, por lo tanto, consideraban encontrarse en una situación de desventaja frente a sus compañeros:

"Yo le digo que me hace falta un material, el profesor le remite al SACU un material, el material en cuestión, el SACU lo amplía y luego voy yo y lo recojo. Este proceso suele tardar un mes, con lo cual los apuntes me llegan más tarde o, en ocasiones, al final del cuatrimestre, por lo que durante un curso tuve que optar por otras cuestiones y alternativas" (Andrés, primera sesión, entrevista biográfica).

Igualmente, señalaron como barrera la necesaria adaptación de los ordenadores de las aulas de informática. Reclamaron que al acceder a dichas instalaciones pudieran sentarse y trabajar como el resto de sus compañeros, que los ordenadores tuvieran las pertinentes elevaciones, con softwares de lectura de pantallas en caso de discapacidad visual, programas específicos de voz, etc ya que, en el caso de Rafa, el no tener los programas adaptados le suponía tener que realizar un trabajo extra en su casa con su ordenador haciendo lo mismo que sus compañeros hacen durante la clase.

\footnotetext{
"Vamos, esos ordenadores están para que la gente los pueda utilizar pero, como yo no puedo utilizarlos, pues un ordenador portátil es lo principal para tú poder manejarte. Porque por ejemplo, si algún ordenador tuviera un programa de voz, pues con la voz lo podría controlar... o un ratón táctil como mi ordenador, también lo podría controlar, lo que pasa es que con los otros ratones no puedo. [...] Con todos los ordenadores que hay, casi ninguno está adaptado" (Rafa, segunda sesión, "Un día en la vida de...").
}

Otro de los aspectos negativos que dos de los estudiantes calificaron como una barrera es el cambio de plan o el plan de Bolonia. Este nuevo plan suponía la extinción de clases y tutorías, siendo un gran obstáculo a su aprendizaje, además de las barreras personales y sociales con las que tuvieron que enfrentarse al tener compañeros diferentes y con edades inferiores. En el caso de Marper, su problema es más específico puesto que se encontró con el obstáculo de secretaría de no reconocer una asignatura que tenía aprobada y que le había supuesto un enorme esfuerzo.

\footnotetext{
“Este año me cambiaron de plan y como aprobé una pues fui a secretaría y poco más, y le... y le pongo de vuelta y media y le dije ‘¿cómo?... cómo no reconozcáis mi aprobado os como a todos. Que ya hace un año que estoy aquí y como no apruebe esta asignatura no puedo continuar, con el esfuerzo que todo esto me ha supuesto"” (Marper, segunda sesión, entrevista focalizada).
}

Con relación a esta queja, Andrés quiso aclarar su disconformidad con el sistema de evaluación implantado a través de los grados, 
con independencia de su discapacidad, ya que aseguraba que se une el sistema de evaluación antiguo con el nuevo, teniendo trabajos, prácticas y exámenes que le restan mucho tiempo de estudio personal y de organización del tiempo para ello y que, en algunas ocasiones, son actividades impuestas que no puntúan para la calificación final pero sí que son obligatorias para poder superar la asignatura.

Otro de los aspectos que este mismo estudiante, Andrés, resalta como negativo es la matrícula ordenada de la universidad, la cual obligaba a tener superadas asignaturas de cursos inferiores para poder matricularse en las de cursos superiores, siendo lo habitual realizarlo con asignaturas de primer curso aunque, en su facultad, lo realizaban con las de primer y segundo curso. Esto le suponía un impedimento para poder obtener los créditos necesarios y optar a las becas que concede el Ministerio de Educación, Cultura y Deporte.

"Y como tenemos matrícula ordenada... Entonces yo me tengo que matricular obligatoriamente de todas las de primero, y para cumplir los requisitos de, por ejemplo, becas del Ministerio, yo tengo que matricularme de un mínimo de asignaturas y tengo también que presentarme también a un mínimo de asignaturas" (Andrés, primera sesión, entrevista biográfica).

En cuanto a las ayudas, Rafa señaló como apoyo y aspecto positivo la reserva de aparcamiento de forma exclusiva para él, así como el apoyo y ayuda recibido por parte del personal de secretaría, quién le instó e informó para realizar la reserva en el parking interno antes que en el externo. Por otro lado, también es valorado de forma muy positiva por parte de los estudiantes el servicio de copistería, puesto que afirmaron que, en la mayoría de las ocasiones, no tenían que esperar cola y le atendían de forma inmediata a su llegada.

“Al principio íbamos a pedir aparcamiento fuera pero nos dijo la secretaría que lo hiciéramos dentro, que teníamos derecho a una plaza de garaje, que también lo veo importante porque aparcamientos no hay nunca y, encima, ahora que han puesto zona azul, más difícil aparcar. Y aparte, los días de lluvia también es importante tener garaje. El garaje significa seguridad, saber que voy a tener un sitio nada más llegar, que tampoco voy a llegar tarde por buscar aparcamiento" (Rafa, segunda sesión, entrevista focalizada).

En referencia al Servicio de Atención a la Comunidad Universitaria (SACU), y más concretamente en relación con el Servicio de Atención a Estudiantes con Discapacidad (SAD), la gran mayoría de los participantes se mostraron satisfechos con la labor que realizan, así como con el personal encargado de atender las necesidades del alumnado con discapacidad. En la medida de sus posibilidades, estos servicios resolvían problemas y ayudaban a cubrir las necesidades de estos estudiantes. Indicaron que la inclusión en el ámbito universitario así como las adaptaciones académicas resultaron más satisfactorias y menos costosas debido a las ayudas ofrecidas por este servicio.

“A la Unidad de Atención a la Discapacidad estoy adscrito como trámite administrativo para facilitar ciertas actuaciones que creo que me conviene llevar a cabo, por ejemplo, me facilita mucho el tema de conseguir lo que son ampliaciones en los exámenes o, por ejemplo, más tiempo para poder seguir el desarrollo de los exámenes al igual que las ampliaciones, o incluso las ayudas de los materiales que solamente están disponibles en soporte impreso... pues yo se lo solicito a esta unidad y ellos lo llevan a cabo" (Andrés, primera sesión).

Unido a lo anterior, se valoró de forma muy positiva la excelente labor realizada por la persona encargada del SAD. Sin embargo, los estudiantes se encontraron insatisfechos con la escasa cobertura que da el servicio, ya que es una sola persona la que debe atender a más de 600 alumnos con discapacidad con los que cuenta la Universidad de Sevilla. Se consideró, por tanto, que son pocos los medios que tiene la universidad para garantizar una ayuda y atención de calidad a toda la comunidad 
universitaria con discapacidad, tal y como hemos indicado anteriormente con el caso de Andrés, y el tiempo estimado de adaptación de los materiales de clase. Además, especificaron que sería necesario que hubiera más personal especializado por materias o áreas y que fueran estos quienes indicaran las adaptaciones pertinentes a las necesidades concretas de las asignaturas.

"Sí que es verdad que en un principio estuve muy bien atendido, me ofrecían los cuadernos autocopiativos" (Andrés, primera sesión).

Hicieron especial hincapié en todas las ayudas que el SACU les brindó: desde la primera toma de contacto en los días previos a la prueba de selectividad, los instrumentos de estudio y trabajo, sus servicios y profesionales, pasando por las libretas auto-copiativas, hasta el alumno colaborador. Estas ayudas las han podido disfrutar todos los estudiantes que han participado en esta investigación, sin embargo, el grado de satisfacción con ellas, tanto en el ámbito académico como profesional, se valoran de distinta manera. En tal caso, Marper destacó como ayuda y apoyo fundamental a su aprendizaje la libreta auto-copiativa para poder tomar apuntes así como el servicio de psicología del SACU, que le proporcionó técnicas de estudio para poder afrontar las asignaturas y la titulación. A esta ayuda se le unía Rafa, quién hizo uso del servicio para enfrentarse a sus problemas personales de timidez y motivación.

"Me dieron la libreta auto-copiativa que fue un gran consuelo, lo más... lo más imprescindible para mí, para tomar los apuntes en el día a día y me han ayudado mucho al desarrollo académico durante todos estos años” (Marper, segunda sesión).

Sin embargo, pese a que el SACU, y en concreto el SAD, se valoró de forma positiva por los estudiantes, en el caso de Andrés hay ayudas como la libreta auto-copiativa o el alumno colaborador que no fueron valoradas de forma positiva y se indicó que no respondían a la demanda real de sus necesidades o a las requeridas por la titulación. Respecto a la libreta auto-copiativa, uno de los inconvenientes que se detalló es la falta de legibilidad de la caligrafía del compañero o, en titulaciones como la suya (Ingeniería), la falta de comprensión del ejercicio que se ha copiado en clase puesto que el compañero lo anotaba y seguía paso a paso las explicaciones del profesor pero, cuando llegaba a manos del estudiante con discapacidad (como es el caso de Andrés), no comprendía lo escrito ni los pasos realizados.

"El tema de los cuadernos auto-copiativos, eso puede ser que funcione en disciplinas de las Ciencias Sociales o incluso en Historia o en ciertas áreas de... si hay a lo mejor un análisis, más concreto, más... sin estructuras muy complejas, que sí se pueden expresar en un cuaderno y que luego tú... o una toma de apuntes, que tú luego puedas leerlo y que luego sea solo memorizarlo y meterlo en la cabeza pues ahí a lo mejor sí funciona; pero eso aplicarlo de manera genérica al resto de las disciplinas no... es poco viable. No se explica lo mismo en una clase de Historia que en una clase de Matemáticas o una clase de Ingeniería. $\mathrm{Si}$, por ejemplo, estás viendo tema de circuito, plantear un circuito en un papel que se calca... y luego haciendo un análisis intenso de eso resulta imposible, porque en realidad lo que te están explicando es cómo alcanzar una solución paso a paso, aquí lo que te están dando es una solución e intenta tú descubrir de dónde ha salido eso" (Andrés, primera sesión).

En el caso del alumno colaborador, estudiantes como Andrés y Rafa mostraron su disconformidad ante tal ayuda, puesto que afirmaron que es una ayuda utópica debido a que no tenían efecto ni beneficio real en sus estudios, y que en numerosas ocasiones el alumno colaborador aceptaba estas ayudas por sus condiciones económicas o que los apuntes facilitados no se entendían. Desde el punto de vista de Andrés, el mayor inconveniente que le encontraba al ayudante colaborador es la repercusión posterior que ejercía sobre la universidad ya que pensaba que, en ocasiones, éste iba a realizar las funciones del profesor y éstos dejaban de tener una mayor y más estrecha relación de colaboración con los estudiantes. 
Por ello, normalmente, tras pasar el primer año de carrera esta ayuda se la ofrecían a un compañero de confianza y que le hubiera estado ayudando sin ningún compromiso, es decir, la ofrecían como un premio puesto que indicaban que aquellos compañeros que realmente querían ayudarlos lo hacían de forma altruista y sin ningún tipo de beneficio a cambio.

En el caso de Rafa, este tipo de ayuda se convertía todavía en más especial puesto que las necesidades que él mostraba no eran sólo académicas sino también físicas, por lo que quedaba a la buena voluntad del alumno colaborador cubrirlas o no. Por ello, reclamaron un tipo de ayuda económica para su madre, que es la persona que le acompañaba en su día a día todas las horas y que está en la universidad. Además, tuvo que abandonar su trabajo para ello por lo que reclamaban recibir una pequeña compensación económica.

"Es que la universidad te pone a alguien para asistirte en lo que es en el ámbito de la educación, que te toma apuntes pero, a la hora de ir al servicio, no te ayuda" (Rafa, segunda sesión).

Otra de las ayudas destacadas por los estudiantes con discapacidad, además de las recibidas por los profesores, por el personal que trabaja en la universidad y por el SACU, es la ayuda recibida por otros organismos sociales independientes de la universidad, como es el caso de la ONCE y de la Junta de Andalucía con el bonobús solidario o la tarjeta de discapacidad, así como el Defensor del Pueblo Andaluz o la Consejería de Bienestar e Igualdad para problemas personales con la universidad y algunos departamentos concretos o recursos y ayudas tecnológicas como un GPS adaptado para personas con discapacidad visual.

“También tengo las ayudas técnicas, únicas y exclusivas que puedan aportar lo que es la ONCE. Yo tengo ayudas técnicas, como pueden ser las lentes ópticas que son unas gafas que mayoritariamente las uso para la lectura del cerca. Tengo también otra ayuda, que es una lupa pero digital que, conectadas a un monitor o a un ordenador, lo que hace es ampliar el texto de la pizarra o un libro que esté cerca de mi” (Andrés, primera sesión).

Aunque, como explicaron estos universitarios, han visto reducidos los recursos y las ayudas debido a los recortes económicos que actualmente sufre nuestro país ya que, tal y como afirma Consuelo, la madre de Rafa, los recursos y herramientas que Rafa puede necesitar para su aprendizaje, como pueden ser un atril, un programa de voz o un ratón inalámbrico, entre otros, tiene que costeárselos de forma personal y privada en ocasiones, no pudiendo ser posible.

\section{Conclusiones y discusión}

Partiendo del objetivo de este artículo, "identificar, describir y explicar las barreras y ayudas que un grupo de estudiantes con discapacidad perciben en universidad como institución”, tratamos de plantear cuáles son las principales barreras y ayudas que los participantes de esta investigación experimentan en la universidad como institución. Para ello, destacaremos cuáles son las claves que han de tener las prácticas inclusivas en la universidad.

Las instituciones de educación superior, en el caso concreto de las personas con discapacidad, suponen una oportunidad y opciones de mejora y empoderamiento de sus propias vidas (Fuller et al., 2004; Moriña, 20I 5; Moriña et al., 20I6; Moswela y Mukhopadhyay, 20I I; Shaw, 2009; Wehman 2006). No obstante, tan sólo el $8 \%$ de este colectivo tiene estudios universitarios. Se trata de un tránsito y experiencia que en ocasiones se ve teñida por las barreras y obstáculos a los que tienen que enfrentarse para aprender y participar en la institución universitaria (Adams y Holland, 2006; Dalmau et al., 2013; Denhart, 2008; Fuller et al.,2004; Gibson, 20I2; Hopkins, 20I I; Vickerman y Blundell, 20го). 
En España, durante el curso 20I4/20I 5, ha habido un descenso en la tasa de matriculación de los estudiantes con discapacidad en la universidad: 21.577 frente a los 21.942 estudiantes matriculados en el curso 20I3/20I 4 (Universia, 20 I 5). Este descenso del I,6\% de estudiantes nos lleva a replantearnos desde nuestro estudio si las declaraciones, normativas e iniciativas existentes -como, por ejemplo, la Convención sobre los Derechos de las Personas con Discapacidad de 2006, las Actas de

Discriminación de países como Australia (I992), Estados Unidos (I990) o Reino Unido (I 995 , 20Iо) o el Real Decreto Legislativo I/20I3 de derechos de las personas con discapacidad y de su inclusión social- se están llevando a cabo en las universidades de forma íntegra y efectiva o si, por el contrario, no son suficientes para garantizar una educación de calidad, sin discriminación, partiendo de los principios de educación inclusiva y del diseño universal de aprendizaje.

Desde nuestro trabajo, los estudiantes que han participado en él no respondieron a este interrogante desde una postura positiva ya que, desde su perspectiva, esta normativa no sólo no era suficiente para garantizar una educación de calidad, sino que también era desconocida por parte de la comunidad universitaria y, por lo tanto, en muchas ocasiones no se ha aplicado. Además, tal y como indicaron los participantes, ésta no resultó eficaz ya que no daba respuesta a los problemas diarios con los que se enfrentaron en la universidad. En este sentido, sería necesario el desarrollo de acciones y medidas para que la normativa existente solventara los problemas con los que actualmente se encuentra nuestro alumnado. Sería interesante, tal y como indican Barnes (2007), Moriña y Molina (201 I), Novo et al. (2015), Forteza (2009) y Valenzuela (2015), la creación de una agenda de trabajo al respecto, el uso de todos los canales y vías de información existentes así como crear políticas de formación y concienciación en las universidades, cuyas acciones se encontraran dirigidas a asegurar los derechos de las personas con discapacidad en educación superior, promoviendo, divulgando y cumpliendo la legislación y garantizando la igualdad de oportunidades, especialmente en los colectivos más vulnerables.
Sería necesario e imprescindible desarrollar planes integrales, creados específicamente para cada persona, en los que se implicara y tuviera cabida la comunidad universitaria (profesores, personal de administración y servicio, y el alumnado). La creación de un profesor tutor podría ser una respuesta a la necesidad de orientación y asesoramiento con la que nuestros estudiantes se encuentran. Además el tránsito de bachillerato a la universidad y el acceso a ésta en sí, para nuestros estudiantes supuso un hito importante en su experiencia, siendo calificado este tránsito como negativo en dos de los casos. Por ello, la creación de una figura de orientación o tutor cuya función fuera guiar, asesorar, motivar y apoyar en el acceso y tránsito a la universidad, informándoles sobre las diversas cuestiones existentes, sería primordial. Además, esta figura podría ser un nexo de unión entre ellos y el profesorado, al informarle sobre su discapacidad y las necesidades educativas que requieren, solventando de esta forma la inadecuada coordinación existente entre la universidad y el profesorado.

Estudios como los de Parker (I999), Vickerman y Blundell (2010), Abad Morrillas et al. (2008), Martínez Usero (2008), Moriña et al. (20I4) o Poussu-Olli (I999), nos hablan de esta figura de apoyo y de la importancia de la misma en los procesos de inclusión de las personas con discapacidad en la universidad. No obstante, nos indican que esta figura únicamente ha de ser un apoyo y que el estudiante nunca ha de ser dependiente del mismo.

Además, uno de los datos reflejados en esta investigación se centró en la ausencia de organismos o entidades que satisficieran sus necesidades durante su estancia en la universidad. Para ello, aunque actualmente existe un servicio de apoyo como es la Unidad de Atención a Estudiantes con Discapacidad, en algunas ocasiones no encontraron respuestas a sus demandas, por lo que se planteó la creación de un servicio de apoyo por facultades o aumentar el alcance de éste dotándolo de más personal. En este sentido, estudios como los de Forteza (2009) y Forteza y Ortego (2003), remarcan la necesidad de replantear 
la estructura funcional y organizacional de los servicios de apoyo con el fin de evaluar a cada estudiante con discapacidad ofreciéndoles los recursos necesarios (Alcantud, I995; Forteza, 2009; Tolrá, 2002).

Otros resultados de esta investigación se centran en el sistema de evaluación que actualmente está implantado a raíz del plan de Bolonia, cuyas premisas se basan en un cambio de paradigma con el aprendizaje del alumno como protagonista (Egido et al., 20I4). Sin embargo, nuestro estudio refleja que para nuestros protagonistas estas premisas no se cumplieron teniendo en ocasiones una carga de trabajo imposible de abarcar. Bajo la perspectiva de estos alumnos, los procesos de evaluación continua a veces son inexistentes, llegando en ocasiones a tener un sistema de evaluación combinado o mixto entre el plan antiguo y extinguido y el actual. Además, el aumento de alumnos en clase, llegando a rozar los casi 60 (o incluso más en algunos casos), lo hace imposible e inabarcable tanto para el profesor como para el alumno. No hay que olvidar tampoco la barrera que supone la obligatoriedad de asistencia a clase.

Otro de los inconvenientes que los estudiantes detectaron derivado del plan de Bolonia es la matrícula ordenada de la universidad, la cual obligaba a tener superadas asignaturas de cursos inferiores para poder matricularse en las de cursos superiores. Además, el no tener todos los créditos superados les impedía e imposibilitaba, al igual que al resto de los compañeros, la concesión de las becas que otorga el Ministerio de Educación, Cultura y Deporte.

Para finalizar con las barreras identificadas en la universidad como institución, los estudiantes hicieron hincapié en la necesaria adaptación de los ordenadores de las aulas de informática. Reclamaron opciones y garantías de igualdad en las instalaciones para poder tener acceso y trabajar como el resto de sus compañeros, con ordenadores en los que tuvieran las pertinentes elevaciones, con softwares de lectura de pantallas en caso de discapacidad visual, programas específicos de voz, etc. En este sentido, encontramos datos similares en trabajos como los de Alcantud et al. (2000) y Castellana y Sala (2005), donde se describen barreras de no adaptación y accesibilidad en los edificios y espacios, o falta de accesibilidad en entornos virtuales (páginas web), entre otros.

En cuanto a las ayudas, los tres estudiantes señalaron como apoyo y aspecto positivo la reserva de aparcamiento de forma exclusiva, así como el apoyo y ayuda recibidos por parte del personal de secretaría, conserjería, copistería y cafetería. No obstante, no nos gustaría terminar este apartado, que cuenta con más barreras que ayudas, sin hacer alusión lo que han supuesto para los estudiantes los estudios universitarios y la universidad en sí, siendo un factor de ayuda y facilitador. Esto les ha ayudado a potenciar el crecimiento personal e intelectual lo que, en la mayoría de los casos, se revierte en una mejora del grado de autonomía y autoestima.

Estos obstáculos nos lleva a pensar, apoyándonos en el modelo social de la discapacidad, que es el contexto el que genera las barreras a las personas con discapacidad y que éstas no son consecuencias de sus barreras personales o individuales, tal y como señalan las premisas del modelo médico de discapacidad. En este sentido, tal y como apuntan Baglieri et al. (20II), Bolt (2004), Dalmau et al. (20I3), Iáñez (2010), Hopkins (20I I) y Jacklin et al. (2007), las dificultades con que los estudiantes con discapacidad se encuentran en la universidad están en su entorno.

Respecto a la Unidad de Atención a Estudiantes con Discapacidad, es importante señalar como algo novedoso y desconocido la información y orientación que se pueda proporcionar para ayudar a poder moverse de manera efectiva dentro de la nueva institución. En nuestro caso, en la universidad, los estudiantes identificaron como principal vínculo de apoyo y de orientación la Unidad de Atención a Estudiantes con Discapacidad, la cual, desde que tienen conocimiento del alumnado con discapacidad y desde su ingreso en la universidad, se pone en contacto para transmitirle toda la información referente a ayudas y becas de las que puedan beneficiarse. 
Castro de Paz y Abad Morillas (2009), Castro de Paz et al. (2006) o Echeita y Verdugo (2005), resaltan en sus trabajos la importancia de la existencia de servicios, comunidades y proyectos de apoyo y orientación al estudiante con discapacidad, coordinando incluso la universidad con el resto de entidades que ofrecen apoyos y ayudas y con la propia Administración en sí. La finalidad de todo este proceso no sería otra que una mayor sensibilización social hacia la inclusión del alumnado universitario con discapacidad (Castro de Paz y Abad Morillas, 2009; Castro de Paz et al., 2006; Sánchez y Carrión, 2010).

Por lo tanto, encontramos que esta Unidad es un gran apoyo al estudiante con discapacidad, ya que desde los primeros días de acceso a la universidad atienden a sus demandas y necesidades, ofreciendo recursos técnicos, adaptaciones curriculares, apoyo personal, etc.

Un número significativo de autores (Abad Morillas et al., 2008; Castellana y Sala, 2005; Castro de Paz y Abad Morillas, 2009; Castro de Paz et al., 2006; Echeíta y Verdugo, 2005; Fraiz Brea et al., 2008; Martínez Usero, 2008; Moriña, 20I0; Moswela y Mukhopadhyay,
20I I; Parker, I999; Poussu-Olli, I999; Sánchez Palomino, 2009, 20II; Vickerman y Blundell, 20I0) defienden este servicio de apoyo al alumnado, destacándolo como imprescindible respecto a la accesibilidad y las prácticas inclusivas en igualdad de oportunidades por parte del alumnado con discapacidad.

Una de las peticiones que dos de los estudiantes realizaron fue el aumento de personal en este servicio, así como herramientas y recursos que dieran respuestas reales a sus necesidades y fueran satisfechas de forma íntegra.

Esta realidad nos lleva a confirmar la necesidad de adaptación y reajuste que se hace evidente en los centros de educación superior para que sean totalmente accesibles para toda la comunidad universitaria. Por ello, tal y como indica Valenzuela (2015), el objetivo que deben de seguir las políticas y los planes de mejora ha de ser alcanzar la inclusión plena, tomando como referencia el diseño universal. En este sentido, Pliner y Johnson (2004) indican que estas mejoras no sólo beneficiarán a los estudiantes con discapacidad sino al colectivo de estudiantes en general. 


\section{Referencias bibliográficas}

Abad Morillas, M. et al. (2008): "Apoyo a la integración con estudiantes con discapacidad en la enseñanza universitaria: Algunas Medidas y Propuestas de actuación orientadora". Educación y Diversidad. Revista InterUniversitaria de Investigación sobre Discapacidad e Interculturalidad, 2: I 29-I 50.

Adams, M., y Holland, S. (2006): Towards Inclusive Learning in higher Education. Developing Curricula for Disabled Students. New York: Rotledge.

Alba, C. y Zubillaga, A. (2O 2): "La utilización de las TICs en la actividad académica de los estudiantes universitarios con discapacidad". Revista complutense de educación, 23 (I): 2350.

Alcantud, F. et al. (2000): La integración de estudiantes con discapacidad en los estudios superiores. Valencia: Universitat de València Estudi General (Servei de Publicacions).

Alcantud, F. (I995): "Estudiantes con discapacidades integrados en los estudios universitarios: notas para su orientación”, en Rivas, F. (ed.): Manual de asesoramiento y orientación vocacional. Madrid: Síntesis.

Baglieri, S. et al. (20I I): "Disability studies and special education: The need for plurality of perspectives on disability". Remedial and Special Education, 32 (4): 267-278.

Barnes, C. (2007): "Citizenship and vulnerability: Disability and issues of social and political engagement”. Brital Journal Sociology, 58 (4): 7I7-718.

Bolt, D. (2004): "Disability and the Rhetoric of Inclusive Higher". Journal of Further Higher Education, 28 (4):353-358.

Borland, J. y James, S. (I 999): “The Learning Experience of Students with Disabilities in Higher Education. A case study of a UK university”. Disability \& Society, I4 (I): 85IOI.
Castellana, M. y Sala, I. (2005): "La Universidad ante la diversidad en el aula". Aula Abierta, 85 : 57-84.

Castro de Paz, J. F. y Abad Morillas, M. (2009): "La incorporación a los estudios superiores: situación del alumnado con discapacidad". Revista Qurriculum, 22: I65-I 88.

Castro de Paz, J. F. et al. (2006): "Universidad y Diversidad: Necesidades Docentes en la atención al alumnado con discapacidad". Revista Qurriculum, I9: I89-209.

Cobos, A. y Moreno, M. (20I4): "Educación superior y discapacidad: análisis desde la experiencia de algunas universidades Colombianas". Revista Española de Discapacidad, 2 (2): 83-IоI.

CRUE y Fundación ONCE (2OI4): Formación curricular en diseño para todas las personas en trabajo social. Madrid: CRUE (en línea). $<$ http://www.fundaciononce.es/sites/default/ files/trabajo_social_ffd4all.pdf $>$, acceso 9 de diciembre de 2016 .

Dalmau, M. et al. (2013): "Formación universitaria e inserción laboral. Titulados españoles con discapacidad y competencias profesionalizadoras”. Revista Española de Discapacidad, I (2): 95-I I 8.

De Coninck, F. y Godard, F. (I998): “El enfoque biográfico a prueba de interpretaciones. Formas temporales de causalidad", en Lulle, T. et al. (coords.): Los usos de la historia de vida en las Ciencias Sociales II. Colombia, AnthroposCIDS.

Denhart, H. (2008): “Deconstructing Barriers: Perceptions of Students Labeled with Learning Disabilities in Higher Education". Journal of Learning Disabilities, 4I (6): 483-497.

Doughty, H. y Allan, J. (2008): “Social capital and the evaluation of inclusiveness in Scottish further education colleges". Journal of Further and Higher Education, 32 (3): 275-284. 
Echeita, G. y Verdugo, M. A. (2005): “Diez años después de la Declaración de Salamanca sobre las necesidades educativas especiales en España. Entre la retórica esperanzadora y las resistencias al cambio". Siglo Cero, 36 (I): 5-I 2.

Egido Gálvez, I. et al. (20I4): "La dimensión social del proceso de Bolonia: Apoyos y servicios para grupos de estudiantes poco representados en las universidades españolas”. Educación XX $X_{I}, I_{7}$ (2): 57-8I.

España. Real Decreto Legislativo I/20I3, de 29 de noviembre por el que se aprueba el Texto Refundido de la Ley General de derechos de las personas con discapacidad y de su inclusión social, Boletín Oficial del Estado, 3 de diciembre de 2013, núm. 289, pp. $95635^{-}$ 95673 .

Ferreira, C. et al. (20I4): "Sistema de indicadores sobre el apoyo a los estudiantes con discapacidad en las universidades españolas”. Revista de Educación, 363: 4I 2-444.

Forteza, D. (2009): “Los nuevos retos para la intervención psicopedagógica en los estudios superiores". Qurriculum: Revista de teoría, investigación y práctica educativa, 22: 35-54.

Forteza, D. y Ortego, J. L. (2003): "Los servicios y programas de apoyo universitarios para personas con discapacidad: estándares de calidad, acción y evaluación”. Revista de Educación Especial, 33: 9-26.

Fraiz Brea, J. et al. (2008): "La accesibilidad como nexo de unión entre la Universidad y la sociedad: la creación de mercados globales e integradores", en De Castro Silva, E. J. y Díaz de Castro, F. J. (coords): Universidad, Sociedad y Mercados Globales. Madrid: Asociación Española de Dirección y Economía de la Empresa (AEDEM).

Fuller, M. et al. (2004): "Incorporating disabled students within an inclusive higher education environment". Disability y Society, I9 (5):455468.

Fundación Universia (2016): Guía de Atención a la Discapacidad en la Universidad (en línea). <http://www.uhu.es/sacu/discapacidad/
doc/ogMari6-SACU-DISCAPACIDADOTRASUNIVERSIDADES-GuiaDeApoyoALas NecesidadesEducativasEeEstudiantesConDisca pacidad.pdf>, acceso 9 de diciembre de 2016 .

Gibson, S. (2OI2): "Narrative accounts of university education: socio-cultural perspectives of students with disabilities". Disability y Society, 27 (3): 353-369.

Goetz J. P. y LeCompte, M. D. (I988): Etnografía $y$ diseño cualitativo en investigación cualitativa. Madrid: Morata.

Goodley, D. et al. (2004): Researching Life Stories: Method, Theory and Analyses in a Biographical Age. London: Routledge Falmer Press.

Guasch, D. y Hernández, J. (20I I): Observatorio Universidad y Discapacidad. Principios de igualdad de oportunidades y Accesibilidad Universal en los Planes de Estudios de los títulos de Grado de las universidades españolas. Barcelona: Observatorio Universidad y Discapacidad.

Hammersley, M. y Atkinson, P. (I994): Etnografía. Métodos de investigación. Barcelona: Paidós.

Holstein, J. y Gubrium, J. (eds.) (20I2): Varities of Narrative Analysis. Thousand Oaks: Sage.

Hopkins, L. (20I I): "The path of least resistance: a voice-relational analysis of disabled students experiences of discrimination in English universities". International Journal of Inclusive Education, I 5 (7): 7 I I-727.

Iáñez, A. (20I0): "Exclusión y Diversidad Funcional. Una propuesta de intervención basada en el modelo de vida independiente". Revista de la Facultad de Trabajo Social, 26 (26): I 2O-I4I.

IMSERSO (2002): Libro verde de la accesibilidad. Madrid: IMSERSO

Jacklin, A. et al. (2007): Improving the experiences of disabled students in higher education (en línea). <http://cascadeoer2.pbworks.com/w/ file/fetch/33757279/jacklin.pdf $>$, acceso I4 de octubre de 20 I I.

Martínez Usero, J. A. (2008): “La Ley 4/2007 de Universidades y la integración de los 
estudiantes con diversidad funcional en la sociedad del conocimiento". Revista de Universidad y Sociedad del Conocimiento, 5 (I): I-9.

McMillan, J. y Schumacher, S. (2005): Investigación educativa. Madrid: Pearson.

Miles, M. B. y Huberman, A. M. (I994): Qualitative data analysis. Beberly Hills, CA: Sage Publications.

Moriña, A. et al. (2016): "What if we could imagine an ideal university? Narratives by students with disabilities". International Journal of Disability Development and Education: I-I 5.

Moriña, A. (201 5): “ ¿Contextos universitarios inclusivos? Un análisis desde la voz del alumnado con discapacidad". Cultura y Educación, 27 (3): 669-694.

Moriña, A. et al. (2015): "What if we could imagine the ideal professor? Proposals for improvement by university students with disabilities". Teaching and Teacher Education, 52: 9I-98.

Moriña, A. et al. (2OI4): "Inclusive curricula in Spanish higher education? Students with disabilities speak out”. Disability \& Society, 29 (I): 44-57.

Moriña, A. y Molina, V. (20I I): "La Universidad a análisis: las voces del alumnado con discapacidad". Revista de Enseñanza Universitaria, 37: 23-35.

Moriña, A. (20I0): "School memories of young people with disabilities: an analysis of barriers and aids to inclusion". Disability \& Society, 25 (2): I63-I 75 .

Moriña, A. (2003): Diversidad en la escuela: Diseño, Desarrollo y Valoración de una propuesta de formación para el profesorado (tesis doctoral). Universidad de Sevilla, Sevilla.

Moswela, E. y Mukhopadhyay, S. (2OII): “Asking for too much? The voices of students with disabilities in Botswana". Disability $y$ and Society, 26 (3): 307-319.

Murray, C. et al. (20I I): "The Effects of DisabilityFocused Training on the Attitudes and
Perceptions of University Staff". Remedial and Special Education, 32 (4): 290-300.

Novo Corti, I. et al. (20I I): “Análisis de las actitudes de los jóvenes universitarios hacia la discapacidad: Un enfoque desde la teoría de la acción razonada”. Relieve, I7 (2): I-26.

Opazo, H. (20II): "Ética en investigación: desde los códigos de conducta hacia la formación del sentido ético". Revista Iberoamericana sobre Calidad, Eficacia y Cambio en Educación, 9 (2): 62-78.

Pacheco, D. (20I2): "Nuevos alcances de la ética en investigación a partir de la ética de la liberación”. Revista Reflexiones, 9I (I): I I 7I 25 .

Parker, V. (I999): "Personal Assistance for Students with Disabilities in HE: The experience of the University of East London”. Disability \& Society, I4 (4): 483-504.

Patton, M. Q. (I987): How to use qualitative methods in evaluation. London: Sage Publications.

Peralta, A. (2007): Libro Blanco sobre universidad $y$ discapacidad. Madrid: Real Patronato sobre Discapacidad.

Pliner, S. y Johnson, J. (2004): "Historical, theoretical, and foundational principles of universal instructional design in higher education". Equity y Excellence in Education, 3: IO5-II3.

Poussu-Olli, H. S. (I999): "To be a Disabled University Student in Finland". Disability \& Society, I4 (I): IO3-II3.

Prowse, S. (2009): "Institutional construction of disabled students". Journal of Higher Education Policy and Management, 3 I (I): 89-96.

Pujadas, J. J. (2002): El método biográfico: El uso de las Historias de Vida en Ciencias Sociales. Madrid: Centro de Investigaciones Sociológicas (edición original, I992).

Pujolà, J. T. (2007): “¿Qué TICs usar con nuestros alumnos de ELE?”, en Jornadas Pedagógicas 2007. Consejería de Educación de la Embajada de España. Estocolmo (Suecia). 
Richaud, M. C. (2007): "La ética en la investigación psicológica”. Enfoques XIX, I (2): 5-I 8 .

Riessman, C. J. (2008): Narrative methods for the human sciences. Thousand Oaks: Sage Publications.

Sánchez, A. y Carrión, J. J. (2010): “Los estudiantes con discapacidad en la Universidad de Almería: Ideas y Actitudes sobre su integración Educativa y Social”. European Journal of Education and Psychology, 3 (3): 329-34I.

Sánchez Palomino, A. (20 I I): "La Universidad de Almería ante la integración educativa y social de los estudiantes con discapacidad: ideas y actitudes del personal docente e investigador". Revista de Educación, 354: 575-603.

Sánchez Palomino, A. (2009): Integración educativa y social de los estudiantes con discapacidad en la Universidad de Almería. Almería: CERMI, Universidad de Almería.

Shaw, J. A. (2009): "Chance happenings in life and psychotherapy". Psychiatry, 72 (I): I-I 2.

Sikes, P. (2006): "On dodgy ground? Problematics and ethics in educational research". International Journal of Research and Method in Education, 29 (I): I05-II 7.

Solla, C. (2013): Guía de buenas prácticas en educación inclusiva. Madrid: Save the Children.

Stutchbury, K. y Fox, A. (2009): "Ethics in educational research: introducing a methodological tool for effective ethical analysis". Cambridge Journal of Education, 39 (4): 489-504.

Tinklin, T. y Hall, J. (I999): "Getting round obstacles: Disabled students' experiences in higher education in Scotland". Studies in

Higher Education, 24 (2): I83-I94.

Tolrá, J. (2002): “Retos de los servicios universitarios de apoyo a la integración”, en VII Reunión sobre Universidad y Discapacidad. Universidad Autónoma de Barcelona (manuscrito no publicado).

Universia (2 de marzo de 20I 5): Disminuye un I, $6 \%$ el número de estudiante con discapacidad matriculados en las universidades españolas (en línea). <http://noticias.universia.es/en-portada/ noticia/20 I 5/03/02/I I 207 I 5/disminuye-I-6-numero-estudiantes-discapacidad-matriculadosuniversidades-espanolas.html>, acceso 7 de agosto de $20 \mathrm{I} 5$.

Vain, P. D. (20I I): “La ética en la investigación educativa y el riesgo del uso de la ciencia como naturalización de lo social". REICE, Revista Electrónica Iberoamericana sobre Calidad, Eficacia y Cambio en Educación, 9 (2): I 5-23.

Valenzuela, A. M. (20 I 5): "Interpelaciones desde la diversidad funcional. La (in) justa medida de la capacidad en Chile". Revista Española de Discapacidad, 3 (2): 55-77.

Vander Kloet, M. (2OI 5): “Accessibility in Teaching Assistant Training: A Critical Review of Programming from Ontario's Teaching and Learning Centres". The CanadianJournal for the Scholarship of Teaching and Learning, 6 (2).

Vickerman, P. y Blundell, M. (2010): “Hearing the voices of disabled students in higher education”. Disability \& Society, 25 (2): 2I-32.

Wehman, P. (2006): Life Beyond the Classroom: Transition Strategies for Young People with Disabilities. Baltimore: Paul H. Brookes Pub. Co. 